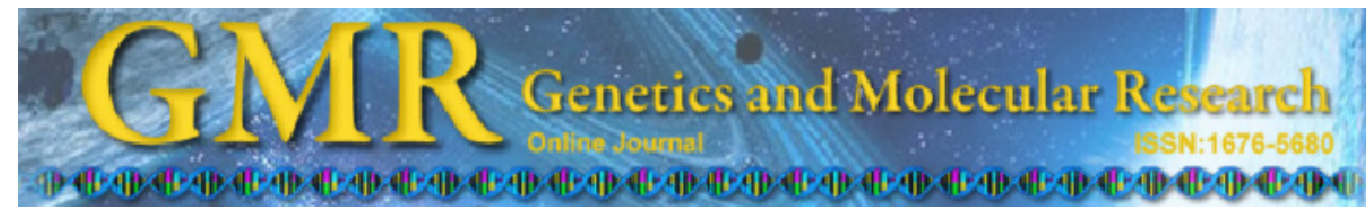

$\underline{\text { Short Communication }}$

\title{
An AFLP-based approach for the identification of sex-linked markers in blunt snout bream, Megalobrama amblycephala (Cyprinidae)
}

\author{
H.O. Rao, J.C. Deng, W.M. Wang and Z.-X. Gao \\ College of Fishery, Key Lab of Agricultural Animal Genetics, \\ Breeding and Reproduction of Ministry of Education/ \\ Key Lab of Freshwater Animal Breeding, Ministry of Agriculture, \\ Huazhong Agricultural University, Wuhan, Hubei, China \\ Corresponding author: Z.-X. Gao \\ E-mail: gaozx@mail.hzau.edu.cn
}

Genet. Mol. Res. 11 (2): 1027-1031 (2012)

Received January 11, 2012

Accepted March 14, 2012

Published April 19, 2012

DOI http://dx.doi.org/10.4238/2012.April.19.7

\begin{abstract}
Sex-specific DNA markers are useful for studying sexdetermination mechanisms and establishment of monosex populations. Three widely spaced geographical populations (Liangzi, Poyang and Yuni Lakes in China) of blunt snout bream (Megalobrama amblycephala) were screened with AFLPs to search for sex-linked markers. Female and male pools (10 individuals in each pool) from each population were screened using 64 different primer combinations. A total of 4789 genomic fragments were produced, with a mean frequency of 75 bands per primer pair. Three different primer combinations produced putative sex-associated amplifications and were selected for individual screening in the three populations. However, none showed sex specificity when we converted these three markers into sequence characterized amplified region markers and evaluated all the individuals from the three populations.
\end{abstract}

Key words: AFLP; Sex-specific marker; SCAR; Megalobrama amblycephala 


\section{INTRODUCTION}

Blunt snout bream (Megalobrama amblycephala Yih, 1955), commonly known as Wuchang fish, is an endemic species in China. Its natural distribution is restricted to the middle and lower reaches of Yangtze River, such as Liangzi (LZ), Poyang (PY) and Yuni (YN) Lakes (Li et al., 1991). M. amblycephala is a species of ray-finned fish in the genus Megalobrama, which includes other three species M. skolkoii, M. hoffmanni and M. pellegrini. Among these four species, M. amblycephala has been widely favored for its delicacy and recognized as a main aquaculture species in the freshwater polyculture system since 1960s in China. As the success of artificial propagation and its high economic value, $M$. amblycephala aquaculture industry has been greatly developed in the past decades. As early as 2001, the total output reached 541,115 tons (CAFS, 2001). However, growth depressions have been reported in the culture stock of $M$. amblycephala due to inbreeding during artificial propagation. Moreover, as its widespread through the transplant and artificial breeding, germplasm resources of the bream were under threat of recession and mixture (Li and Yang, 1996). Nowadays, the cultured M. amblycephala is gradually exhibiting early sexual maturity, low growth rate and susceptible to disease. By comparison, early sexual maturity of female M. amblycephala can result in reduced feeding and growth as reported in other fish species (Simpson et al., 1996). Monosex male populations can eliminate the problem of precocious female maturity and their consequences (Dunham, 1990). Therefore, sex control has important application potential in M. amblycephala aquaculture. Such monosex populations are typically produced by sex reversal and selective breeding. The generation and maintenance of monosex stocks require that genetic and phenotypic sex can be independently discernible.

The molecular marker technique has been demonstrated to be an effective tool for both the identification of sex-specific genetic markers and sex control (Liu and Cordes, 2004). Amplified fragment length polymorphism (AFLP) analysis in combination with bulked segregant analysis (BSA) approach has been shown to be a fast and efficient way to identify markers linked to target genes responsible for desirable traits (Michelmore et al., 1991). AFLP screening with BSA approach was used successfully in the isolation of sex-specific markers of aquatic animals including the Nile tilapia Oreochromis niloticus (Ezaz et al., 2004), the rainbow trout Oncorhynchus mykiss (Felip et al., 2005), and the spotted halibut Verasper variegatus (Ma et al., 2010).

In the present study, the AFLP technique was used to identify sex-specific markers in M. amblycephala based on pooled DNA samples from known male and female individuals, which were sampled from its three natural populations.

\section{MATERIAL AND METHODS}

M. amblycephala were collected from three wild populations in Liangzi, Yuni and Poyang Lakes (Table 1). Caudal fin samples were taken from 20 mature individuals (10 females and 10 males) of each population. The phenotypic sex of fish was determined by distinguishing external morphology during the reproductive season or gonadal structure of the histological section. Total genomic DNA was extracted from $50 \mathrm{mg}$ tissue sample according to Waters et al. (2000). The quality and concentration of DNA was measured by NanoDrop 2000 and then adjusted to $100 \mathrm{ng} / \mu \mathrm{L}$. 


Table 1. Samples and geographic information of sampling sites.
\begin{tabular}{llcccc}
\hline Name & Locality & Area $\left(\mathrm{km}^{2}\right)$ & Latitude & Longitude & No. of samples \\
\hline Liangzi Lake & Hubei Province & 227.15 & 30.34 & 114.51 & 20 \\
Yuni Lake & Hubei Province & 152.1 & 29.85 & 112.12 & 20 \\
Poyang Lake & Jiangxi Province & 2262 & 29.24 & 115.95 & 20 \\
\hline
\end{tabular}

AFLP technique in combination with BSA approach was performed as previously described (Michelmore et al., 1991; Vos et al., 1995; Ezaz et al., 2004) with minor modification. Genomic DNAs of female and male from each population were pooled to generate female $(\mathrm{N}=$ $10 \mathrm{fish}$ ) and male ( $\mathrm{N}=10 \mathrm{fish}$ ) pools. Six pooled DNA samples were prepared using $10 \mu \mathrm{L}$ (100 $\mathrm{ng} / \mu \mathrm{L})$ genomic DNA from each individual. Each pool (500 ng) was digested with EcoRI/MseI prior to ligation with restriction site-specific adaptors (Table 2). Pre-amplication was carried out utilizing adaptor common primers, and selective amplification by 64 different primer combinations (Table 2) was conducted with diluted (50-fold) pre-amplification product. AFLP-PCR products were separated on $6 \%$ denaturing polyacrylamide gels and visualized by silver staining.

Table 2. The AFLP adapters, common primers and selective primer used in this study for sex-specific marker screening in Megalobrama amblycephala.

\begin{tabular}{lll}
\hline \multirow{2}{*}{ Primer } & \multicolumn{2}{c}{ Primer sequence (from 5' to 3' direction) } \\
\cline { 2 - 3 } & EcoRI & MseI \\
\hline Adapter & CTC GTA GAC TGC GTA CC & GAC GAT GAG TCC TGA G \\
Common primer & E00: GAC TGC GTA CCA ATT C & M00: GAT GAG TCC TGA GTA A \\
Selective primer & E1: E00+AAC & M1: M00+CAT \\
& E2: E00+AAG & M2: M00+CAA \\
& E3: E00+ACA & M3: M00+CCA \\
& E4: E00+ACT & M4: M00+CCT \\
& E5: E00+AGA & M5: M00+CTA \\
& E6: E00+AGC & M6: M00+CTT \\
& E7: E00+ATC & M7: M00+CGA \\
& E8: E00+ATG & M8: M00+CGC \\
\hline
\end{tabular}

Sex-specific bands were directly excised from the $6 \%$ denaturing polyacrylamide gel and eluted from the gel by incubation in $30 \mu \mathrm{L}$ sterilizing water at $4^{\circ} \mathrm{C}$ overnight. The re-amplification PCR was performed with the corresponding primer pairs under the same conditions as before. PCR products were recovered from agarose gels using gel extraction kit and cloned into PMD-18 T vector as described (Ma et al., 2010) and then selected for sequencing. The sequences of different clones of the same marker and the sequences of the different markers were assembled and compared using the DNAMAN version 4.0 software. Based on the sequence information, the primer pairs were designed with the Primer Premier 5.0 software and synthesized commercially (Sangon, Shanghai, China). SCAR primers were employed to amplify the genomic DNA of the female and male individuals from three populations.

\section{RESULTS AND DISCUSSION}

A total of 4789 legible genomic fragments were identified with an average of 75 bands per primer combination. Based on the primary screens across the pooled DNA samples, 30 , 28 and 21 different primer combinations, which gave putative sex-associated amplifications, 
were selected for individual screening in different geographical populations. Based on the analysis of the individual DNA samples, three primer combinations showed sex-associated bands in different populations (Table 3). However, when we converted the three markers into SCAR markers, none showed a different band pattern between female and male individuals from three populations.

$\begin{aligned} & \text { Table 3. Summary of putative sex-linked markers and their percent in females and males Megalobrama } \\
& \text { amblycephala, respectively. }\end{aligned}$
\begin{tabular}{llccc} 
Primer combinations & Populations & Size & $\begin{array}{c}\text { Sex of DNA pools with } \\
\text { sex-specific bands }\end{array}$ & $\begin{array}{c}\text { Percent of individuals with } \\
\text { sex-specific bands }\end{array}$ \\
\hline M7/E7 & Poyang Lake & $846 \mathrm{bp}$ & Male & $70 \%$ \\
M2/E1 & Yuni Lake & $830 \mathrm{bp}$ & Male & $90 \%$ \\
& Poyang Lake & & Male & $50 \%$ \\
M5/E5 & Yuni Lake & $886 \mathrm{bp}$ & Female & $80 \%$ \\
& Poyang Lake & & Female & $40 \%$ \\
\hline
\end{tabular}

Recently, AFLP screening and BSA were used successfully in the isolation of sexspecific markers of aquatic animals. However, many studies have demonstrated that sexspecific markers could vary among species, strains or even geographical populations. The female-specific marker identified by Xia et al. (2011) in loach Paramisgurnus dabryanus from the ancient Yellow River Wetland did not show sex specificity in the closely related species Misgurnus anguillicaudatus and other two geographical populations. Chen et al. (2009) demonstrated that the male-specific markers observed in the common carp Cyprinus carpio from the Yellow River did not show sex specificity in three other common carp populations. Other sex-linked markers found in the Antarctic icefish Chionodraco hamatus (Capriglione et al., 1994) and the piapara semen Leporinus elongatus (Nakayama et al., 1994) both show variation among strains. These studies suggest that sex-specific markers may vary among strains and geographical populations owing to the DNA mutation, insertion or deletion events that occur on or near the sex-specific sites (Gao et al., 2010). In the present study, the AFLP fragments that produced by M2/E1 and M5/E5 showed a significant difference between females and males when evaluated in the individual females and males from related populations. But, none showed sex specificity when we converted the AFLP markers into SCAR markers.

The study to identify sex-specific markers in fish species seems laborious owing to the variability and plasticity in sex chromosome organization. The success of the identification of sex-specific markers in fish species is relevant with the presence of a sex chromosome or non-chromosomal genetic sex-determining mechanisms in the target species (Sriphairoj et al., 2007). In M. amblycephala, sex chromosomes have not been identified by classical karyotype (Lu et al., 1984). In addition, sex determination mechanism and sex-linked markers have not been studied in M. amblycephala in the published literature. The failure in search of sex-specific DNA markers could be due to the size of the genome, the number of markers screened and the proportion of the genome that is sex specific in the species studied (Penman and Piferrer, 2008). Our failure to identify sex-specific markers seems to strengthen the possibility that sex chromosomes are either not present or they weakly differentiated in the genomes of $M$. amblycephala.

Our study was the first attempt, to our knowledge, to find sex-specific markers in $M$. amblycephala. Despite the failure to find such markers, our data provide some useful information for further studies targeting similar goals. 


\section{ACKNOWLEDGMENTS}

Research supported by grants from the Modern Agroindustry Technology Research System entitled "Staple Freshwater Fishery Industry Technology System" (\#CARS-46-05) and the Fundamental Research Funds for the Central Universities (\#2011PY023 and \#2011PY043).

\section{REFERENCES}

CAFS (2001). Fishery Statistic Data. Chinese Academy of Fishery Sciences, Beijing.

Capriglione T, Morescalchi A, Olmo E, Rocco L, et al. (1994). Satellite DNAs, heterochromatin and sex chromosomes in Chionodraco hamatus (Channichthyidae, Perciformes). Polar Biol. 14: 285-290.

Chen JJ, Wang YL, Yue YY, Xia XX, et al. (2009). A novel male-specific DNA sequence in the common carp, Cyprinus carpio. Mol. Cell. Probes 23: 235-239.

Dunham RA (1990). Production of monosex or sterile fish in aquaculture. CRC. Rev. Aquat. Sci. 2: 1-17.

Ezaz MT, Harvey SC, Boonphakdee C, Teale AJ, et al. (2004). Isolation and physical mapping of sex-linked AFLP markers in nile tilapia (Oreochromis niloticus L.). Mar. Biotechnol. 6: 435-445.

Felip A, Young WP, Wheeler PA and Thorgaard GH (2005). An AFLP-based approach for the identification of sex-linked markers in rainbow trout (Oncorhynchus mykiss). Aquaculture 247: 35-43.

Gao ZX, Wang HP, Yao H and Wang WM (2010). No sex-specific markers detected in bluegill sunfish Lepomis macrochirus by AFLP. J. Fish. Biol. 76: 408-414.

Li S and Yang X (1996). Effects of two-way selection on biochemical genetics of blunt snout bream (Megalobrama amblycephala). J. Fish. Sci. China 1: 1-5.

Li S, Cai W and Zhou B (1991). Morphological and biochemical genetic variations among populations of blunt snout bream (Megalobrama amblycephala). J. Fish. China 15: 204-211.

Liu ZJ and Cordes JF (2004). DNA marker technologies and their applications in aquaculture genetics. Aquaculture 238: 1-37.

Lu RH, Li YJ and Xu KS (1984). A chromosome study of Megalobrama amblycephala Yih. Oceanol. Limnol. Sin. 15: 487-493.

Ma HY, Chen SL, Yang JF, Ji XS, et al. (2010). Isolation of sex-specific AFLP markers in spotted halibut (Verasper variegatus). Environ. Biol. Fish. 88: 9-14.

Michelmore RW, Paran I and Kesseli RV (1991). Identification of markers linked to disease-resistance genes by bulked segregant analysis: a rapid method to detect markers in specific genomic regions by using segregating populations. Proc. Natl. Acad. Sci. U. S. A. 88: 9828-9832.

Nakayama I, Foresti F, Tewari R, Schartl M, et al. (1994). Sex chromosome polymorphism and heterogametic males revealed by two cloned DNA probes in the ZW/ZZ fish Leporinus elongatus. Chromosoma 103: 31-39.

Penman DJ and Piferrer F (2008). Fish gonadogenesis. Part I: genetic and environmental mechanisms of sex determination. Rev. Fish. Sci. 16: 16-34.

Simpson AL, Metcalfe NB, Huntingford FA and Thorpe JE (1996). Pronounced seasonal differences in appetite in Atlantic salmon parr, Salmo salar: effects of nutritional state and life history strategy. Funct. Ecol. 10: 760-767.

Sriphairoj K, Na-Nakorn U, Brunelli JP and Thorgaard GH (2007). No AFLP sex-specific markers detected in Pangasianodon gigas and P. hypophthalmus. Aquaculture 273: 739-743.

Vos P, Hogers R, Bleeker M, Reijans M, et al. (1995). AFLP: a new technique for DNA fingerprinting. Nucleic Acids Res. 23: 4407-4414.

Waters JM, Epifanio JM, Gunter T and Brown BL (2000). Homing behavior facilitates subtle genetic differentiation among river populations of Alosa sapidissima: microsatellites and mtDNA. J. Fish. Biol. 56: 622-636.

Xia X, Zhao J, Du Q, Zhi J, et al. (2011). Cloning and identification of a female-specific DNA marker in Paramisgurnus dabryanus. Fish Physiol. Biochem. 37: 53-59. 